\title{
Disgust and Contamination Sensitivity in Vaginismus and Dyspareunia
}

\author{
Peter J. de Jong · Mark van Overveld • \\ Willibrord Weijmar Schultz • Madelon L. Peters • \\ Femke M. Buwalda
}

Received: 1 August 2006/Revised: 8 May 2007/Accepted: 5 June 2007/Published online: 2 October 2007

(C) Springer Science+Business Media, LLC 2007

\begin{abstract}
This study examined the potential role of disgust propensity and contamination sensitivity in vaginismus. Women suffering from vaginismus $(n=20)$ or dyspareunia $(n=22)$, and a group of women without sexual complaints $(n=30)$ completed self report measures indexing their (1) general dispositional disgust propensity, and (2) sensitivity for (ideational) contamination by sexual stimuli as a function of its source (self, partner, unknown). In support of the idea that disgust may be involved in vaginismus, women with vaginistic complaints displayed a generally enhanced dispositional disgust propensity. The sensitivity for contamination by sexual stimuli did not vary across groups. However, especially when the source was the participant's partner, the willingness ratings might have been influenced by demand and may, therefore, not accurately reflect participant's actual sensitivity for contamination by sexual stimuli. Future studies using more implicit or behavioral measures are necessary to more definitely test the role of disgust in vaginismus.
\end{abstract}

Keywords Disgust - Contamination · Vaginismus · Dyspareunia $\cdot$ Sexual dysfunctions

P. J. de Jong $(\bowtie)$. F. M. Buwalda

Department of Clinical and Developmental Psychology,

University of Groningen, Grote Kruisstraat 2/1, Groningen 9712

TS, The Netherlands

e-mail: p.j.de.jong@rug.nl

M. van Overveld · M. L. Peters

Department of Clinical Psychological Science, Maastricht

University, Maastricht, The Netherlands

W. Weijmar Schultz

Department of Obstetrics \& Gynecology, University Medical

Centre Groningen, University of Groningen, Groningen,

The Netherlands

\section{Introduction}

Vaginismus is defined as a recurrent or persistent involuntary spasm of the musculature of the outer third of the vagina that interferes with intercourse (American Psychiatric Association, 2000). The vaginistic complaints are characterized by persistent difficulties in allowing vaginal entry of the penis, a finger, and/or object, despite the woman's expressed wish to do so (Basson et al., 2003). These complaints often have a chronic course and may result in considerable emotional distress (e.g., ter Kuile et al., 2007). Unfortunately, the etiology of this "perplexing condition" is largely unknown (Leiblum, 2000) and currently available treatment strategies are not very effective in reducing these complaints (e.g., van Lankveld et al., 2006).

For a long time, the dominant view implied that vaginismus essentially reflects a medical problem, and much effort has been invested in designing and testing various surgical solutions for the impossibility to have sexual intercourse (Abromov, Wolman, \& David, 1994). More recently, injection with botuline (perhaps better known as Botox) is applied to paralyze the pelvic floor muscles to allow penetration (Ghazizadeh \& Nikzad, 2004; Münchau \& Bhatia, 2000).

Current psychological explanations imply highly aversive sexual experiences and/or sexual harassment as an important factor in the etiology of vaginismus (Rathus, Nevid, \& Fichner-Rathus, 2005). Accordingly, the DSM-IV$T R$ (American Psychiatric Association, 2000) refers to sexual trauma as an etiological feature of vaginismus. However, recent empirical studies showed that sexual trauma is neither a necessary nor a sufficient condition for the generation of vaginistic complaints. Although a considerable proportion of women suffering from vaginismus report a history of sexual abuse (in terms of attempts of sexual abuse and/or 
forced sexually touching or being touched with hands, mouth or objects) (e.g., Reissing, Binik, Khalifé, Cohen, \& Amsel, 2003), there are also a considerable number of women suffering from these complaints who indicate that they have not experienced such abuse (e.g., ter Kuile et al., 2007). The specificity of a history of sexual abuse in the etiology of vaginismus is further questioned by the finding that sexual abuse is also quite frequent in women who suffer from complaints that certainly do not involve difficulties to allow vaginal entry, such as sexual addiction (Carnes, 1998). Hence, it appears that this type of specific aversive conditioning experiences is not relatively frequent in women suffering from vaginistic complaints.

More recently, it has been argued that pain-related fears may be critically involved in vaginismus (e.g., Reissing, Yitzchak, Khalifé, Cohen, \& Amsel, 2004). Although the report of pain is not a requirement for the diagnosis of vaginismus, women with vaginismus often suffer from comorbid pain complaints (Reissing et al., 2004). Accordingly, it has been shown that a considerable percentage of women with lifelong vaginismus report vestibular pain on touch with a cotton swab (ter Kuile, van Lankveld, Vliet Vlieland, Willekes, \& Weijenborg, 2005). Following on from this, one could argue that vaginistic reactions may reflect a defensive response that is elicited by fear of pain associated with penetration.

Another promising candidate that may help improving the conceptualization of vaginismus is disgust and contamination sensitivity (e.g., de Jong \& Peters, in press). Psychological views of sexual behavior roughly consider sexual dysfunction as a consequence of a negative emotional reaction to erotic stimulation (e.g., Barlow, 1986; Janssen \& Everaerd, 1993). Although disgust seems an obvious candidate of being one of these negative emotional reactions interfering with healthy sexual behavior and/or sexual pleasure, current theories and empirical research predominantly focus on emotional and cognitive processes related to fear and pain (e.g., Payne, Binik, Amsel, \& Khalifé, 2005), whereas the reference to disgust is mainly anecdotal (e.g., Carnes, 1998; Kaneko, 2001). Therefore, the present study was designed as a first step to more systematically examine the role of disgust in vaginismus.

From an evolutionary perspective, disgust is seen as a defensive mechanism protecting the organism from contamination by pathogens (Rozin \& Fallon, 1987). Accordingly, disgust is focused on the intersection between the body and the environment and concentrates on the skin and body apertures (Fessler \& Haley, 2006; Rozin, Nemeroff, Horowitz, Gordon, \& Voet, 1995). The strength of the disgust response increases as a function of proximity of the potential contaminant and the sense of inclusion. It has been found that, for women, the vagina is the body part with the highest contamination sensitivity, whereas the penis of an unfamiliar male has the highest contamination potency (Rozin et al., 1995). Given the central role of these organs in the context of sexual behavior, together with the fact that bodily products (e.g., saliva, sweat, semen) and smells are among the strongest disgust elicitors (Rozin \& Fallon, 1987), it is not very difficult to envisage that feelings of disgust and disgustrelated appraisals may arise during sex, which, in turn, may inadvertently influence sexual behaviors. There is also some empirical evidence showing that sexual stimuli indeed are capable of evoking feelings of disgust (e.g., Carnes, 1998; Koukounas \& McCabe, 1997). In addition, some clinical cases have been described in the literature, in which sexual stimuli seemed to elicit such profound feelings of disgust, that these people even attempt to avoid anything sexual in themselves and others, a condition known as "sexual anorexia" (Carnes, 1998).

It is highly conceivable that disgust and fear of contamination elicit defensive reflexes that may interfere with functional sexual behaviors. There is evidence that involuntary contraction of the pelvic floor muscles is part of a general defense mechanism (van der Velde, Laan, \& Everaerd, 2001) that may be elicited by (the anticipation of) fearful and/or painful occurrences (e.g., van der Velde \& Everaerd, 2001). It seems reasonable to assume that similar defensive circumvaginal contractions can be elicited or potentiated by disgust-related appraisals (e.g., Yartz \& Hawk, 2002). Following this, the prospect of mere physical contact with the vagina (a highly contamination sensitive body part) and/or the anticipation of penetration by the partner's penis (a body part with very high contamination potency; Rozin et al., 1995) may well elicit involuntary pelvic floor muscle activity (cf. van der Velde \& Everaerd, 2001). From this perspective, the difficulty of penetration in women suffering from vaginismus may at least partly be due to a disgustinduced defensive response.

In addition to so-called core disgust, animal-reminder disgust may also play a role. In their two-stage model of disgust, Rozin, Haidt, and McCauley (1999) argued that the defensive mechanism of disgust originally evolved to prevent the body from contamination by pathogens and toxins from the outside environment (core disgust) is extended to stimuli and/or behaviors that remind us of our animal nature. This disgust-mediated rejection of our animal nature is argued to serve a defensive function by maintaining the hierarchical division between humans and animals via distancing the self from animals and animal properties (Haidt, McCauley, \& Rozin, 1994). Since sexual behavior is highly suggestive of our underlying animal nature, sexual behaviors and/or sexual advances may well elicit disgust to guard the human-animal border and may thus give rise to avoidance behaviors (e.g., defensive circumvaginal contractions) interfering with functional sexual behaviors. 
So both core disgust and animal-reminder disgust may well contribute to problems with having sexual intercourse in women suffering from vaginismus. Insight into the possible role of (core and animal reminder) disgust in vaginismus may help improving the conceptualization of this disorder. This may not only be of theoretical interest, but may also provide important new clues for improving available interventions. To explore the possible role of disgust in vaginismus, the present study was designed to examine whether women with vaginismus are characterized by relatively high levels of disgust propensity. High levels of disgust propensity for stimuli from the domain of core-disgust elicitors are assumed to enlarge the probability of particular stimuli (e.g., a penis or sperm) to acquire high contamination potency. In addition, high disgust propensity is assumed to increase the likelihood that particular body parts (e.g., the vagina) acquire relatively high contamination sensitivity (cf. Davey, Forster, \& Mayhew, 1993; de Jong, Andrea, \& Muris, 1997). Both characteristics will logically facilitate the generation of disgust motivated avoidance tendencies, such as the contraction of the pelvic musculature at the prospect of penetration. In a similar vein, high disgust propensity for stimuli of the animal-reminder type is likely to increase the probability that sexual behaviors and/or organs acquire disgust-evoking properties. So if disgust plays an important role in vaginismus, high levels of disgust propensity would set women at risk for developing vaginistic complaints.

For a proper appreciation of the role of disgust in vaginismus, it would be important to have insight into the type of disgust-related preoccupations that are most prominently involved. Therefore, we measured both the disgust propensity for stimuli from the domain of core and animalreminder elicitors. The present study sought also to investigate more directly the disgust-evoking properties of sexual stimuli in connection with vaginismus. Disgusting stimuli share the crucial feature that they readily transfer their offensive characteristics to other stimuli by brief contact, even when there is no detectable trace of the contaminant (e.g., Rozin \& Fallon, 1987). In this study, we examined to what extent sexual stimuli share this feature of all disgusting objects, and whether there are differences between women with and without vaginismus in this respect. Previous work in the context of spider phobia showed that a hypothetical (i.e., in vitro) behavioral test revealed similar results as an actual behavioral test (e.g., de Jong, Vorage, \& van den Hout, 2000). For practical reasons, we therefore used in this study an in vitro rather than an actual behavioral test.

To test the specificity of enhanced disgust propensity and contamination sensitivity in women suffering from vaginismus, we added both a control group of women without sexual complaints, and a clinical control group of women suffering from dyspareunia, a sexual dysfunction from the same diagnostic category as vaginismus (i.e., sexual pain disorders). Whereas the inability to have sexual intercourse is most central to vaginismus, for dyspareunia recurrent genital pain is the defining feature (American Psychiatric Association, 2000). Although disgust-related preoccupations might also contribute to dyspareunia, current views emphasize the role of fearful preoccupations with painful intercourse in the maintenance of dyspareunia (e.g., Reissing et al., 2004). Therefore, we anticipated that disgust and contamination sensitivity would be most relevant for women suffering from vaginismus.

\section{Method}

Participants

During two 3-month periods, all women who applied for treatment at the Department of Gynecology of the Academic Medical Center of Groningen and were clinically diagnosed as having primary (life-long) vaginismus ( $n=20$; M age $=30.2$ years, $\mathrm{SD}=6.1$, range $=20-39$ years $)$ or dyspareunia $(n=22$; $\mathrm{M}$ age $=24.9$ years; $\mathrm{SD}=4.1$, range $=19-35$ years $)$ were included in the present study. Mean educational level was 9.3 $(\mathrm{SD}=1.2)$ for the vaginismus group and $8.8(\mathrm{SD}=1.3)$ for the dyspareunia group on a scale ranging from 0 (no education completed) to 11 (Master's degree). A control group of women without sexual complaints but within the same age range and level of education was recruited via advertisements in local newspapers asking for healthy, sexually active women without sexual complaints in the age range from 18 to 40 years, who were willing to volunteer in research on the mechanisms involved in vaginism (explained as the inability to have sexual intercourse $)(n=30 ; \mathrm{M}$ age $=26$ years; $\mathrm{SD}=3.1$, range $=$ 22-33 years; $\mathrm{M}$ level of education $=9.6, \mathrm{SD}=0.9$ ).

The level of education was similar for all groups, $F(2,68)$ $n s$, but the groups differed with respect to their mean age, $F(2,68)=9.13, p<.05$. Post hoc comparisons indicated that women with vaginismus were significantly older than both the dyspareunia and control group ( $p s<.01)$.

All participants were involved in a heterosexual relationship for at least 3 months. More detailed information concerning the aim and procedure of the present study was provided to all participants as part of the informed consent procedure. The study was approved by the Medical Ethical Committee of the University of Groningen. Women in both clinical groups were diagnosed by an experienced gynecologist/sexuologist using a semi-structured sexual interview. The diagnostic procedure also included a physical examination. According to the DSM-IV-TR (American Psychiatric Association, 2000), vaginismus is characterized by persistent, involuntary spasms of vaginal muscles that interfere with intercourse. However, since relevant studies could not demonstrate the presence of vaginal spasms, it appears that the hallmark of vaginismus is more accurately defined as a 
problem with penetration which extends to sexual intercourse (e.g., Binik et al., 2002). In line with this, it has been proposed to modify and extend the original diagnosis of vaginismus into: "persistent or recurrent difficulties to allow vaginal entry of a penis, a finger, and/or any object, despite the women's expressed wish to do so, whereas structural or physical abnormalities were ruled out during the physical examination" (Basson et al., 2003). In this study, the diagnosis of vaginismus was made on the basis of these criteria formulated by Basson et al. The women with dyspareunia were characterized by persistent or recurrent pain with attempted or complete vaginal entry and/or penile vaginal intercourse. Complaints of dyspareunia should have been present in minimally $50 \%$ of the intercourse attempts for at least 6 months. Exclusion criteria for women with dyspareunia were vulvar pain not directly related to intercourse and lifelong vaginismus causes of dyspareunia. A diagnosis of vulvar vestibulitis syndrome (VVS) was not an exclusion criterion since the etiology of VVS is largely unknown (Lotery, McClure, \& Galask, 2004).

\section{Measures}

\section{General Disgust Propensity}

To assess general (trait) disgust propensity, participants completed the Disgust Scale (DS; de Jong \& Merckelbach, 1998; Haidt et al., 1994). The DS is a widely used and validated 32-item self-report index of disgust and contamination sensitivity covering seven domains of disgust elicitors: Food (e.g., "You are about to drink a glass of milk when you smell it is spoiled"), Animals (e.g., "You are walking barefoot on concrete and you step on an earthworm"), Body Products (e.g., "You see a bowel movement left unflushed in a public toilet"), Sex (e.g., "You hear about an adult woman who has sex with her father"), Envelope Violation (e.g., "You see a man with his intestines exposed after an accident"), Death (e.g., "You accidentally touch the ashes of a person who has been cremated"), and Hygiene (e.g., "I never let any part of my body touch the toilet seat in public restrooms"). In addition, there is an eighth scale referring to the domain of Magical Thinking (Magic). This scale reflects the sympathetic magic laws of contagion (e.g., "Even if I was hungry, I would not drink a bowl of my favorite soup if it had been stirred by a brand-new flyswatter") and similarity (e.g., "A friend offers you a piece of chocolate shaped like dog-doo"). The magical thinking subscale cuts across the seven domains of disgust elicitors and seem to guide individuals' disgust rejections irrespective of the domain. Heightened sensitivity to these magic laws is assumed to enhance disgust responses even in the absence of actual contaminating potency, and to hamper extinction (for a more detailed discussion of these laws, see
Rozin \& Fallon, 1987). Following Haidt et al. (1994), the Food, Animal, and Body Product subscales are interpreted to reflect core-disgust, whereas the other four scales (Sex, Envelope Violations, Death, and Hygiene) are taken to reflect animal reminder disgust. Each scale contains 4 items. The DS includes two true-false and two disgust-rating items for each of the eight domains. The disgust rating items were scored on a 3 point scale: $0=$ "not disgusting at all," $0.5=$ "slightly disgusting," and 1 = "very disgusting." Scores were summed and yield a total score between 0 and 32 .

\section{Contamination Potency of Sexual Products}

To investigate individual differences with respect to the level of the contamination potency of sex-related stimuli, we used a self-report measure, the Sexual Disgust Questionnaire (SDQ), that was specifically designed for the present study. The SDQ was based on the Disgust Questionnaire (DQ) which was originally designed by Rozin, Fallon, and Mandell (1984) to assess food rejection tendencies following trace (or pure ideational) contamination of these food items by certain animal products. The original DQ consisted of items such as: "Imagine your favorite soup. How much would you like to eat this soup after it has been stirred by a used fly swatter?" This questionnaire was modified in a way to test the strength of individuals' tendencies to avoid physical contact with certain objects (e.g., a towel) after it has been in contact with certain sexual stimuli (e.g., sperm of their partner). Since the contamination potency of particular sexual stimuli may vary as a function of the source of the potential contaminants, we differentiated among three possible sources: an unknown person, their partner, and the participant. The SDQ consisted of 9 items (3 for each source; see Appendix). For each item, participants were asked to indicate their willingness to perform a certain action that implied physical contact with certain sexual stimuli on a scale ranging from 0 (certainly not willing) to 8 (certainly willing). For each source, mean scores were subjected to the analyses. Psychometric analysis of the SDQ in a previous study among university students and employees of Maastricht University $(n=458)$ supported the a priori factor structure (Genten, 2005). In addition, the test-retest reliability was high $(r=.92)$. The internal consistency of the total scale was also high (Cronbach's alpha $=0.90$ ), whereas the Cronbach's alphas of the subscales ranged from 0.64 to 0.66 which is just below the range that is regarded as satisfactory for comparing groups (0.7-0.8; Bland \& Altman, 1997). In the present study, Cronbach's alphas for the subscales were 0.62 (self), 0.65 (unknown), and 0.50 (partner). Because the internal consistencies were relatively low (especially for the partner items) differential results of the subscales should be interpreted with care. 


\section{Results}

\section{General Disgust Sensitivity}

The DS total scores as well as the scores on the various subscales are summarized in Table 1 as a function of group.

First, DS total scores (means of all items) were subjected to an one-way ANOVA. Results showed a significant main effect of Group, $F(2,69)=6.66, p<.005, \eta^{2}=.16$. Post hoc comparisons indicated that the scores of the vaginismus group were significantly higher than those of the dyspareunia group, $p<.05, \eta^{2}=.13$, and those of the healthy controls, $p<.005$, $\eta^{2}=.22$. There was no significant difference between the dyspareunia and the control groups $\left(\eta^{2}=.01\right)$.

To examine potentially domain specific differences in (trait) disgust propensity among groups, mean DS scores of the relevant subscales were subjected to a 2 (Domain) $\times 3$ (Group) ANOVA with the first factor being a within subjects factor. The ANOVA showed a main effect of Domain, $F(1$, $69)=12.97, p<.001, \eta^{2}=.16$, indicating that, in line with previous studies, disgust propensity scores were generally higher for the domain of core disgust elicitors than for the animal reminder disgust elicitors (see Table 1). There was a significant main effect of Group, $F(2,69)=6.45, p<.005$, $\eta^{2}=.16$. In line with predictions, Bonferroni controlled post hoc tests indicated that the scores of the vaginismus group were significantly higher than those of the control group, $p=.002, \eta^{2}=.23$. The difference between both patient groups was marginally significant, $p=.06, \eta^{2}=.11$, whereas the difference between the dyspareunia patients and healthy controls were not significant $\left(\eta^{2}=.02\right)$.

Finally, the Magic subscale was subjected to an one-way ANOVA. The ANOVA showed a significant main effect of Group, $F(2,69)=3.17, p<.05, \eta^{2}=.15$. Post hoc comparisons indicated that the score of the vaginismus group was significantly higher than that of the dyspareunia group, $p<.05, \eta^{2}=.11$, whereas a similar tendency was evident between the vaginismus group and the healthy controls, $p=.08, \eta^{2}=.09$. The scores of the dyspareunia group and of the healthy controls did not differ significantly.

To test whether the high level of disgust propensity in the vaginismus group may be due to their relatively high age, we computed post hoc a Pearson correlation between age and DS total score for the women with vaginismus. This correlation was negative and nonsignificant $(r=-.11)$.

\section{Contamination Potency of Sexual Products}

Mean scores on the SDQ are shown in Table 1 as a function of the source of contamination. To test whether vaginistic women attributed relatively strong contaminating properties

Table 1 Mean scores on all measures as a function of group

\begin{tabular}{|c|c|c|c|c|c|c|}
\hline \multirow[t]{3}{*}{ Measures } & \multicolumn{6}{|c|}{ Group } \\
\hline & \multicolumn{2}{|c|}{ Vaginismus $(n=20)$} & \multicolumn{2}{|c|}{ Dyspareunia $(n=22)$} & \multicolumn{2}{|c|}{ Controls $(n=30)$} \\
\hline & M & $\mathrm{SD}$ & M & SD & M & SD \\
\hline \multicolumn{7}{|l|}{ Disgust propensity } \\
\hline Disgust Scale (total) & 20.3 & $4.5^{\mathrm{a}}$ & 16.8 & $4.8^{\mathrm{b}}$ & 15.8 & $3.9^{\mathrm{b}}$ \\
\hline Core & 2.7 & $0.6^{\mathrm{a}}$ & 2.4 & 0.7 & 2.1 & $0.6^{\mathrm{b}}$ \\
\hline Food & 2.1 & $0.9^{\mathrm{a}}$ & 1.8 & 1.0 & 1.4 & $0.8^{\mathrm{b}}$ \\
\hline Animal & 2.8 & 1.0 & 2.8 & 0.9 & 2.4 & 0.8 \\
\hline Body products & 3.1 & $0.8^{\mathrm{a}}$ & 2.5 & 1.0 & 2.7 & $0.9^{\mathrm{b}}$ \\
\hline Animal-reminder & 2.5 & $0.6^{\mathrm{a}}$ & 2.0 & $0.6^{\mathrm{b}}$ & 1.9 & $0.6^{\mathrm{b}}$ \\
\hline Sex & 2.8 & $0.7^{\mathrm{a}}$ & 2.5 & $0.4^{\mathrm{b}}$ & 2.5 & $0.5^{\mathrm{b}}$ \\
\hline Hygiene & 1.9 & $0.8^{\mathrm{a}}$ & 1.4 & 0.8 & 1.3 & $0.8^{\mathrm{b}}$ \\
\hline Envelope violations & 3.2 & 0.8 & 2.5 & 0.8 & 2.8 & 1.0 \\
\hline Death & 2.2 & $1.1^{\mathrm{a}}$ & 1.7 & 0.9 & 1.2 & $0.9^{\mathrm{b}}$ \\
\hline Magic & 2.2 & $1.2^{\mathrm{a}}$ & 1.5 & 0.9 & 1.6 & $0.7^{\mathrm{b}}$ \\
\hline \multicolumn{7}{|c|}{ Contamination potency of sexual products ${ }^{1}$} \\
\hline \multicolumn{7}{|l|}{ Source of contamination } \\
\hline Self & 5.1 & 2.0 & 4.8 & 1.9 & 5.3 & 1.7 \\
\hline Partner & 5.7 & 1.7 & 5.6 & 1.3 & 5.8 & 1.2 \\
\hline Unknown & 2.1 & $1.6^{\mathrm{a}}$ & 2.9 & 1.8 & 3.0 & $1.6^{\mathrm{b}}$ \\
\hline
\end{tabular}

1 Low scores reflect low willingness to tolerate physical contact with stimuli that are potentially "contaminated" by sexual stimuli. For each source, the scale ranged from 0 to 8 . Scores with different superscripts differ significantly from each other as indicated by Bonferroni controlled post hoc tests 
Table 2 Pearson correlations between Disgust Propensity (DS) and the contamination potency of sexual products

\begin{tabular}{llll}
\hline & DS-total & DS-core & $\begin{array}{l}\text { DS-animal- } \\
\text { reminder }\end{array}$ \\
\hline Contamination-Self & -.26 & -.21 & -.22 \\
Contamination-Partner & -.16 & -.17 & -.11 \\
Contamination-Unknown & $-.48^{* *}$ & $-.45^{* *}$ & $-.42^{* *}$ \\
\hline
\end{tabular}

For all correlations, $n=72$

$* * p<.01 ; * p<.05$ (Bonferroni corrected for multiple correlations)

to sex-related products and to explore whether the anticipated contaminating properties of such stimuli varied as a function of the contaminant's source (self, partner, unknown person), SDQ-contamination scores were subjected to a 3 (Group) $\times 3$ (Source) ANOVA with the second factor being a within subjects factor. The ANOVA showed a significant main effect of Source, $F(2,138)=134.80, p<.001$, $\eta^{2}=.66$. The Source by Group interaction was not significant, $F(4,138)=1.54, p=.19, \eta^{2}=.04$. Pairwise comparisons indicated that the contaminating potency of sexual products was significantly stronger when the contaminant's source was unknown compared to when the source was the participant herself or her partner (both $p s<.001$ ). In addition, the contaminating potency was overall significantly stronger when the participant herself was the contaminant's source than when her partner was the source of potential contamination $(p<.001)$.

Disgust Propensity and Avoidance of Physical Contact with Sex-Related Stimuli

To investigate further the relationship between participants' general disgust propensity and their self-reported tendency to avoid physical contact with sex-related stimuli, Pearson correlations were computed (see Table 2). As can be seen in Table 2, DS scores were significantly associated with avoidance of physical contact with sexual stimuli when an unknown person was the source of potential contamination. Although the relationship between DS scores and avoidance of sex-related stimuli was in the same direction when the partner or the participant herself was the source of potential contamination, these correlations did not reach the conventional level of significance. Overall, the pattern of correlations was similar for core and animal-reminder disgust-propensity.

\section{Discussion}

The present study represents a first attempt to explore the role of disgust propensity and contamination sensitivity in two types of sexual dysfunction. The major results can be summarized as follows: First, women with vaginistic complaints showed heightened levels of disgust propensity compared to both women with dyspareunia and women without complaints, whereas no systematic differences were evident between women with dyspareunia and complaint free control women. Second, disgust propensity in women suffering from vaginismus was similarly enhanced for animal-reminder and core disgust elicitors. Third, no convincing evidence emerged to indicate that women with vaginismus (or dyspareunia) were characterized by an enhanced avoidance of physical contact with sexual products.

\section{Disgust Propensity}

In line with predictions, the results indicate that patients with vaginistic complaints were characterized by relatively high levels of general disgust propensity. The heightened disgust propensity in women suffering from vaginismus appeared not restricted to core disgust elicitors but was similarly enhanced for elicitors from the animal-reminder domain. This pattern of findings points to the possibility that core and animal-reminder disgust are both somehow involved in vaginismus. In addition, women with vaginismus showed relatively high scores on the Magic subscale, indicating that these women were relatively sensitive to the sympathetic magic laws of contagion ("once in contact, always in contact") and similarity (Haidt et al., 1994). Heightened sensitivity to these magic laws will lower the threshold for stimuli to acquire a disgust-evoking status even in the absence of actual contaminating potency, and will hamper extinction (Rozin \& Fallon, 1987). Both characteristics are likely to facilitate the generation of relatively persistent and "irrational" disgust-induced avoidance behaviors (e.g., elicited by "innocent" physical contact of the vagina).

The relationship between (core) disgust propensity and vaginismus may be explained by assuming that intercourserelated stimuli (e.g., penis) are more likely to acquire inflated contamination potency and/or that the vagina is more likely to acquire inflated contamination sensitivity in women with high than in women with low levels of (core) disgust propensity (cf. Davey et al., 1993). Both possibilities will logically facilitate the generation of disgust motivated avoidance tendencies, such as the contraction of the pelvic musculature at the prospect of penetration, and/or at the prospect of potential contaminants making mere physical contact with contamination sensitive body parts, such as the vagina. The relationship between animal-reminder disgust propensity and vaginismus may be explained by assuming that body parts and behavioral activities that are related to basic biological functions and remind us of our animal nature are more likely to acquire disgust-eliciting properties (Rozin et al., 1999) in women with high than in women with low 
levels of animal-reminder disgust propensity. Heightened disgust evoking properties of sexual organs and/or behaviors, in turn, may add to problems with vaginal entry.

It should be acknowledged, however, that the finding that women with vaginismus displayed enhanced levels of disgust propensity does not necessarily imply that disgust per se is directly related to the generation of vaginistic complaints. On the basis of the present study, it cannot be ruled out that the relation between vaginismus and disgust propensity is mediated by some other yet unknown factor. Future studies that focus more directly on the disgustevoking properties of sexual stimuli and behaviors are necessary to gain further insight in how exactly disgust, as well as disgust induced defensive reflexes, may play a role in the generation of vaginistic complaints. As a first attempt to more directly examine the disgusting status of sexual stimuli, the present study explored the contamination potency of particular sexual products (e.g., sperm).

\section{Contamination Potency of Sexual Stimuli}

To investigate individual differences with respect to the level of the contamination potency of sex-related stimuli, we based our measurement on the central feature of disgusting stimuli, namely, that they readily transfer their offensive characteristics to other stimuli by brief contact, even when there is no detectable trace of the contaminant (e.g., Rozin \& Fallon, 1987). Following on from this, we asked participants to indicate their willingness to tolerate physical contact with a stimulus (e.g., a towel) that had been in contact with sexual products (e.g., sperm). The self-reported willingness to tolerate physical contact with such stimuli that were potentially contaminated by sexual products was lowest when the source of these products was an unknown person, and highest when the source was their partner.

In apparent conflict with the notion that core disgust plays an important role in vaginismus, the willingness ratings were similar for all groups. However, especially when the source was the participant's partner, the willingness ratings might have been influenced by demand and may, therefore, not accurately reflect participants' actual sensitivity for contamination by sexual stimuli. The finding that only the SDQscores referring to the unfamiliar person were associated with participants' ratings on the Disgust Scale (see Table 2) but not those referring to the partner or self, also points in this direction. In addition, it might be that the positive appreciation of their partners has counteracted participants' initial avoidance tendencies. The present finding that participants generally reported stronger contamination potency for sexual products of themselves than of their partners adds to the possibility that indeed this type of consideration might have played a role here. Moreover, it should be acknowledged that, in the present sample, the internal consistency of the SDQ was rather low. This was especially the case for the items referring to the participant's partner being the source of contamination. Therefore, the present results suggesting that enhanced contamination sensitivity for sexual products does not play a role in vaginismus should be interpreted with care. To arrive at more final conclusions concerning the possible role of enhanced contamination potency of sexual stimuli in vaginismus, it would be important to improve the psychometric properties of the SDQ.

In addition, it would be important for future studies to use more implicit measures of contamination potency that cannot be influenced by deliberate considerations (e.g., facial EMG). The use of implicit measures, such as the Implicit Association Task (IAT; Greenwald, McGhee, \& Schwartz, 1998), might also be worthwhile to assess automatic associations that are not necessarily accessible to introspection. Automatically elicited associations are assumed to play a crucial role in guiding relatively spontaneous, uncontrollable behaviors, whereas more deliberate reflective considerations may be more important for more controllable approach/avoidance behaviors (Huijding \& de Jong, 2006; Strack \& Deutsch, 2004). Accordingly, it might well be that uncontrollable, automatically activated associations are responsible for the contraction of the circumvaginal musculature at the prospect of penetration, whereas the more deliberate associations guide the wish for having intercourse with the partner (cf. Gheldof, de Jong, Vinck, \& Houben, 2004). If, indeed, automatic contamination related associations are critically involved in vaginismus, this may also help explaining why the vaginomuscular contraction is experienced as an automatic and uncontrollable response.

Some comments are in order with respect to the limitations of the present study. First, it should be acknowledged that, although the age range was quite similar for all groups of women, the mean age of the women with vaginistic complaints was higher than that of both other groups of women. Following this, one could speculate that the relatively high level of disgust propensity in the women with vaginismus may be due to their relatively high age. However, this seems not very likely because, in adults, disgust propensity tends to decrease rather than to increase with age (Fessler \& Navarette, 2005). Consistent with this, post hoc analysis within the present group of women with vaginismus showed that, if anything, there was a negative correlation between age and disgust propensity. Therefore, it seems safe to conclude that the relatively high disgust propensity in women with vaginismus does not simply reflect age differences across groups.

Second, to index the contamination potency of sexual stimuli the present study used self-report measures rather than actual behavioral tasks. Although previous studies have shown that the self reported tendency to avoid contact with 
particular disgusting stimuli is strongly related to actual avoidance tendencies as measured during behavioral tasks (e.g., Rozin, Haidt, McCauley, Dunlop, \& Ashmore, 1999), it cannot be ruled out that in the particular domain of sexual stimuli, self-report measures are relatively sensitive to selfpresentational concerns and other influences that may threaten the validity of self-reports as an index of the contamination potency of sexual stimuli. Following this, it would be important to cross-validate the present study using behavioral tasks.

Third, it should be acknowledged that the measure that was used to more directly examine disgust towards sexual stimuli (i.e., SDQ) covered only a limited number of sexual products. Meanwhile, it might well be that the contamination potency of sexual products greatly varies as a function of the particular sexual stimuli. In addition, the subjective contamination potency may vary as a function of context and motivational set (cf. Seelig, 1930). This may also help explaining the relatively low internal consistency of the SDQ. So it seems important for future research to add more items to the present SDQ to more accurately cover all relevant sexual stimuli in various contexts that may play a role here. Fourth, the SDQ was only relevant for contaminationrelated preoccupations (i.e., core disgust). It would be important for future research to include measures that are also sensitive to animal-reminder related preoccupations with sexual stimuli, as both types of disgust may be involved in vaginismus.

To conclude, the present study provided preliminary empirical support for the idea that disgust propensity is somehow involved in the generation of vaginistic complaints. Future studies that focus more directly on the disgust-evoking properties of sexual stimuli are necessary to gain further insight in how exactly disgust, as well as disgust induced defensive reflexes, may play a role in the generation of vaginistic complaints.

Acknowledgments The authors are grateful to Dirma Ter Heege, Marije Schoonbeek, Jördis Trautmann, Esther Sportel, and Esther Canrinus for their assistance during data acquisition.

\section{Appendix}

\section{Sexual Disgust Questionnaire (SDQ)}

In this questionnaire, you will be asked to imagine a number of situations. Please indicate for each of the situations to what extent you would be willing to carry out the described behavior on a scale ranging from 0 to 8 . Cross the number next to each statement that best reflects your judgment when the scale is defined as follows: $0=$ certainly not willing, $2=$ presumably not willing, $4=$ perhaps willing, $6=$ presumably willing, 8 certainly willing.

\begin{tabular}{lccccccc}
\hline $\begin{array}{l}\text { Certainly } \\
\text { not } \\
\text { willing }\end{array}$ & $\begin{array}{l}\text { Presumably } \\
\text { not } \\
\text { willing }\end{array}$ & $\begin{array}{l}\text { Perhaps } \\
\text { willing }\end{array}$ & $\begin{array}{l}\text { Presumably } \\
\text { willing }\end{array}$ & $\begin{array}{l}\text { Certainly } \\
\text { willing }\end{array}$ \\
\hline 0 & 1 & 2 & 3 & 4 & 5 & 6 & 7 \\
\hline
\end{tabular}

To what extent are you willing to:

1. Use a towel for your face that has been thoroughly cleaned after it has been used to wipe off sperm of your partner after sexual intercourse.

2. Use a towel for your face after it has been used to wipe off vaginal fluids from yourself after sexual intercourse and smells accordingly.

3. To lie beneath bedclothes, under which you just had sexual intercourse with your partner.

4. Use a towel for your face after it has been used to wipe off sperm from your partner after sexual intercourse and smells accordingly.

5. To lie beneath bedclothes in a hotel, that look unwashed and below which previous guests may have had sexual intercourse.

6. Use a towel for your face that has been thoroughly cleaned after it has been used to wipe off vaginal fluids of yourself after sexual intercourse.

7. To lie beneath bedclothes below which you have masturbated the day before and which show obvious smudges.

8. Use a towel for your face that has been thoroughly cleaned after it has been used following sexual intercourse to wipe off sperm/vaginal fluid of an unknown person (e.g., a towel in a hotel).

9. To touch a soiled, unwashed towel, that is possibly used to wipe off sperm/vaginal fluid of an unknown person after sexual intercourse (e.g., a towel in a hotel).

\section{References}

Abromov, L., Wolman, I., \& David, M. P. (1994). Vaginismus: An important factor in the evaluation and management of vulvar vestibulitis syndrome. Gynecologic and Obstetric Investigation, 38, 194-197.

American Psychiatric Association. (2000). Diagnostic and statistical manual of mental disorders (4th ed., text rev.). Washington, DC: Author.

Barlow, D. H. (1986). The causes of sexual dysfunction: The role of anxiety and cognitive interference. Journal of Consulting and Clinical Psychology, 54, 140-148.

Basson, R., Leiblum, S., Brotto, L., Derogatis, L., Foucroy, J., FuglMeyer, K., et al. (2003). Definitions of women's sexual dysfunction reconsidered: Advocating expansion and revision. Journal of Psychosomatic Obstetrics and Gynecology, 34, 221-229.

Binik, Y. M., Reissing, E. D., Pukall, C., Flory, N., Payne, K. A., \& Khalifé, S. M. D. (2002). The female sexual pain disorders: Genital pain or sexual dysfunction? Archives of Sexual Behavior, 31, 425-429.

Bland, J. M., \& Altman, D. G. (1997). Statistics notes: Cronbach's alpha. British Medical Journal, 314, 572. 
Carnes, P. J. (1998). The case for sexual anorexia: An interim report on 144 patients with sexual disorders. Sexual Addiction \& Compulsivity, 5, 293-309.

Davey, G. C. L., Forster, L., \& Mayhew, G. (1993). Familial resemblances in disgust sensitivity and animal phobias. Behaviour Research and Therapy, 31, 41-50.

de Jong, P. J., Andrea, H., \& Muris, P. (1997). Spider phobia in children: Disgust and fear before and after treatment. Behaviour Research and Therapy, 35, 559-562.

de Jong, P. J., \& Merckelbach, H. (1998). Blood-injection-injury phobia and fear of spiders: Domain specific individual differences in disgust sensitivity. Personality and Individual Differences, 24, 153-158.

de Jong, P. J., \& Peters, M. L. (in press). Sex and the sexual dysfunctions in women: The role of disgust and contamination sensitivity. In B. Olatunji \& D. McKay (Eds.), Disgust and its disorders. Washington, DC: American Psychological Association.

de Jong, P. J., Vorage, I., \& van den Hout, M. A. (2000). Counterconditioning in the treatment of spider phobia: Effects on disgust, fear, and valence. Behaviour Research and Therapy, 38, 1055-1069.

Fessler, D. M. T., \& Haley, K. J. (2006). Guarding the perimeter: The outside-inside dichotomy in disgust and bodily experience. Cognition and Emotion, 20, 3-19.

Fessler, D. M. T., \& Navarette, C. D. (2005). The effect of age on death disgust: Challenges to terror management perspectives. Evolutionary Psychology, 3, 279-296.

Genten, M. H. (2005). Psychometric quality of the Sexual Disgust Questionnaire $(S D Q)$. Unpublished master's thesis, University of Maastricht, Maastricht, the Netherlands.

Ghazizadeh, S., \& Nikzad, M. (2004). Botulinum toxin in the treatment of refractory vaginismus. Obstetrics and Gynecology, 104, 922-925.

Gheldof, E. L. M., de Jong, P. J., Vinck, J., \& Houben, R. M. A. (2004). Attitudes toward physical activity: The role of implicit versus explicit associations. In G. J. G. Asmundson, J. W. S. Vlaeyen, \& G. Crombez (Eds.), Understanding and treating fear of pain (pp. 117-137). Oxford: Oxford University Press.

Greenwald, A. G., McGhee, D. E., \& Schwartz, J. L. K. (1998). Measuring individual differences in implicit cognition: The Implicit Association Test. Journal of Personality and Social Psychology, 74, 1464-1480.

Haidt, J., McCauley, C., \& Rozin, P. (1994). Individual differences in sensitivity to disgust: A scale sampling seven domains of disgust elicitors. Personality and Individual Differences, 16, 701-713.

Huijding, J., \& de Jong, P. J. (2006). Specific predictive power of automatic spider-related affective associations for controllable and uncontrollable fear responses. Behaviour Research and Therapy, $44,161-176$.

Janssen, E., \& Everaerd, W. (1993). Determinants of male sexual arousal. Annual Review of Sex Research, 4, 211-245.

Kaneko, K. (2001). Penetration disorder: Dyspareunia exists on the extension of vaginismus. Journal of Sex \& Marital Therapy, 27, $153-155$.

Koukounas, E., \& McCabe, M. (1997). Sexual and emotional variables influencing sexual response to erotica. Behaviour Research and Therapy, 35, 221-231.

Leiblum, S. R. (2000). Vaginismus: A most perplexing problem. In S. R. Leiblum \& R. C. Rosen (Eds.), Principles and practice of sex therapy (3rd ed., pp. 181-202). New York: Guilford Press.

Lotery, H. E., McClure, N., \& Galask, R. P. (2004). Vulvodynia. Lancet, 363, 1058-1060.

Münchau, A., \& Bhatia, K. P. (2000). Uses of botulinum toxin injection in medicine today. British Medical Journal, 320, 161-165.
Payne, K. A., Binik, Y. M., Amsel, R., \& Khalifé, S. (2005). When sex hurts, anxiety and fear orient attention towards pain. European Journal of Pain, 9, 427-436.

Rathus, S. A., Nevid, J. S., \& Fichner-Rathus, L. (2005). Human sexuality in a world of diversity. Boston: Pearson Education.

Reissing, E. D., Binik, Y. M., Khalifé, S. M. D., Cohen, D. M. D., \& Amsel, R. M. A. (2003). Etiological correlates of vaginismus: Sexual and physical abuse, sexual knowledge, sexual self-schemata, and relationship adjustment. Journal of Sex and Marital Therapy, 29, 47-59.

Reissing E. D., Yitzchak B. M., Khalifé S. M. D., Cohen D. M. D., \& Amsel, R. M. A. (2004). Vaginal spasm, pain and behavior: An empirical investigation of the diagnosis of vaginismus. Archives of Sexual Behavior, 33, 5-17.

Rozin, P., \& Fallon, A. E. (1987). A perspective on disgust. Psychological Review, 94, 23-41.

Rozin, P., Fallon, A. E., \& Mandell, R. (1984). Family resemblance in attitudes to food. Developmental Psychology, 20, 309-314.

Rozin, P., Haidt, J., \& McCauley, C. R. (1999). Disgust: The body and soul emotion. In T. Dalgleish \& M. Power (Eds.), Handbook of cognition and emotion (pp. 429-445). Chichester, England: Wiley.

Rozin, P., Haidt, J., McCauley, C., Dunlop, L., \& Ashmore, M. (1999). Individual differences in disgust sensitivity: Comparisons and evaluations of paper-and-pencil versus behavioral measures. Journal of Research in Personality, 33, 330-351.

Rozin, P., Nemeroff, C., Horowitz, M., Gordon, B., \& Voet, W. (1995). The borders of the self: Contamination sensitivity and potency of the body apertures and other body parts. Journal of Research in Personality, 29, 318-340.

Seelig, E. (1930). Die Ambivalenz der Gefühle im Zuge des Sexualerlebens [The ambivalence of the feelings in sexual experience]. Zeitschrift für Angewandte Psychologie, 36, 138-150.

Strack, F., \& Deutsch, R. (2004). Reflective and impulsive determinants of social behavior. Personality and Social Psychology Review, 3, 220-247.

ter Kuile, M. M., van Lankveld, J. J. D. M., Vliet Vlieland, C., Willekes, C., \& Weijenborg, P. Th. M. (2005). Vulvar vestibulitis syndrome: An important factor in the evaluation of lifelong vaginismus? Journal of Psychosomatic Obstetrics \& Gynecology, 26, 245-249.

ter Kuile, M. M., van Lankveld, J. J. D. M., de Groot, E., Melles, R., Neffs, J., \& Zandbergen, M. (2007). Cognitive-behavioral therapy for women with lifelong vaginismus: Process and prognostic factors. Behaviour Research and Therapy, 45, 359-373.

van Lankveld. J. J. D. M., ter Kuile, M. M., de Groot, H. E., Melles, R., Nefs, J., \& Zandbergen, M. (2006). Cognitive-behavioral therapy for women with lifelong vaginismus: A randomized waiting-list controlled trial of efficacy. Journal of Consulting and Clinical Psychology, 74, 168-178.

van der Velde, J., \& Everaerd, W. (2001). The relationship between involuntary pelvic floor muscle activity, muscle awareness and experienced threat in women with and without vaginismus. Behaviour Research and Therapy, 39, 395-408.

van der Velde, J., Laan, E., \& Everaerd, W. (2001). Vaginismus, a component of a general defensive reaction. An investigation of pelvic floor muscle activity during exposure to emotion-inducing film excerpts in women with and without vaginismus. International Urogynecology Journal and Pelvic Floor Dysfunction, 12, 328-331.

Yartz, A. R., \& Hawk, L. W. (2002). Addressing the specificity of affective startle modulation: Fear versus disgust. Biological Psychology, 59, 55-68. 